\title{
MGD-GAN: Text-to-Pedestrian generation through Multi-Grained Discrimination
}

\author{
Shengyu Zhang ${ }^{\dagger}$, Donghui Wang ${ }^{\dagger}$, Zhou Zhao ${ }^{\dagger}$, Siliang Tang ${ }^{\dagger}$, Di Xie Xei Wu $^{\dagger}$ \\ ${ }^{\dagger}$ College of Computer Science and Technology, Zhejiang University, China \\ ${ }^{\star}$ Hikvision Research Institute, China \\ \{sy_zhang, dhwang, zhaozhou, siliang, wufei\}@zju.edu.cn, xiedi@ hikvision.com
}

\begin{abstract}
In this paper, we investigate the problem of text-to-pedestrian synthesis, which has many potential applications in art, design, and video surveillance. Existing methods for text-tobird/flower synthesis are still far from solving this finegrained image generation problem, due to the complex structure and heterogeneous appearance that the pedestrians naturally take on. To this end, we propose the Multi-Grained Discrimination enhanced Generative Adversarial Network, that capitalizes a human-part-based Discriminator (HPD) and a self-cross-attended (SCA) global Discriminator in order to capture the coherence of the complex body structure. A fined-grained word-level attention mechanism is employed in the HPD module to enforce diversified appearance and vivid details. In addition, two pedestrian generation metrics, named Pose Score and Pose Variance, are devised to evaluate the generation quality and diversity, respectively. We conduct extensive experiments and ablation studies on the caption-annotated pedestrian dataset, CUHK Person Description Dataset. The substantial improvement over the various metrics demonstrates the efficacy of MGD-GAN on the textto-pedestrian synthesis scenario.
\end{abstract}

\section{Introduction}

Synthesizing visually authentic images from textual descriptions is a representative topic of creative AI systems. It requires a high-level understanding of natural language descriptions, usually vague and incomplete, and the imaginative ability in order to draw visual scenes. Recently, methods based on deep generative models, e.g., Generative Adversarial Networks (Goodfellow et al. 2014), have accomplished promising outcomes in this field. With the generation quality increasing, more challenging objectives are expected, i.e., fine-grained image generation( $(\mathrm{Wei}, \mathrm{Wu}$, and Cui 2019).

Fine-grained image generation, as the term implies, focuses on fine-grained categories, such as faces of a certain individual or objects within a subcategory. Existing methods for fined-grained text-to-image generation only focuses on birds and flowers (Reed et al. 2016, Zhang et al. 2017; $\mathrm{Xu}$ et al. 2018; Zhu et al. 2019a; Qiao et al. 2019). However, text-to-pedestrian generation is a new research direc-

Copyright (C) 2020, Association for the Advancement of Artificial Intelligence (www.aaai.org). All rights reserved.

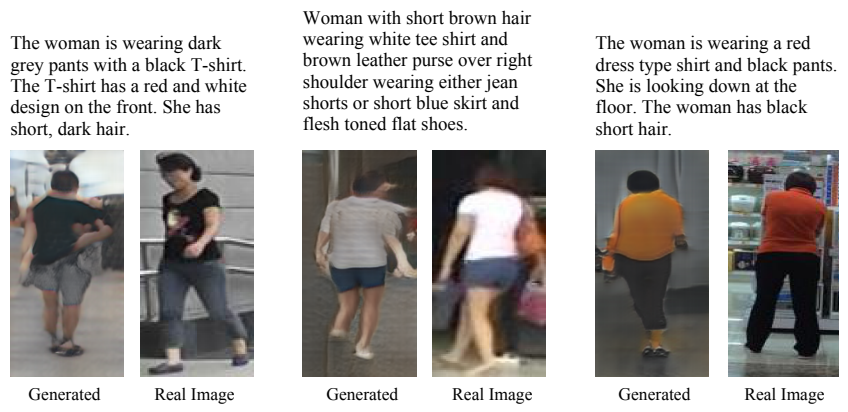

Figure 1: Examples generated by our proposed MGD-GAN.

tion and has many potential applications in movie making (Ma et al. 2017), art creation, and video surveillance - for instance, the appropriate generation of one suspect's portrait according to a witness or victim descriptions. We have carried out an experiment to demonstrate that pedestrian generation can significantly improve the text-to-person search results. Also, text-to-pedestrian generation is a rather difficult problem due to the nature of larger intra-class variances in person generation. The intra-class variances can be summarized as the inter-person variances, such as gender, age, height, and the intra-person variances, such as appearance variances, pose variances.

Not surprisingly, existing methods that are designed for a general text-to-image synthesis problem are still far from solving the pedestrian generation problem. On the one hand, existing methods fail to leverage the task-specific prior knowledge in the pedestrian generation process. To this end, we investigate the person re-id techniques and propose a human-part-based Discriminator, named HPD, which independently penalizes imperfect structure at each human part. On the other hand, as stated above, we view text-topedestrian generation as a fined-grained generation problem. Although there are works (Xu et al. 2018; Zhu et al. 2019a) that exploits a fine-grained word-level attention mechanism in the generation process, the absence of fine-grained discrimination may induce an unbalanced competition between the Generator and Discriminator since they have different levels of abilities. In essence, the Generator focuses on more 
fine-grained local details while the Discriminator can only capture the global coherence of the image.

To handle the problems above, we enhance the humanpart-based Discriminator with a Visual-Semantic Attention module, named VISA. The difference of a regular conditional discriminator and a VISA enhanced discriminator is that rather than signifies the concatenated input of a fake image and a sentence "real" or "fake", whereas the VISA enhanced discriminator scores the importance of each word for each image region and signifies each region "real" or "fake" concerning different words. This setting can help discriminator better penalize the inconsistency between textual descriptions and generated images in a words-regions level, which makes VISA-HPD a local discriminator. As a counterpart of the local one, we construct another self-crossattended global discriminator functioning at the sentenceimage level, penalizing irregular body structure as a whole.

To the best of our knowledge, our work is the initiative to do text-to-pedestrian synthesis. The most commonly used evaluation metric for the generic image generation, the Inception Score, relies on a classification model. However, there are no specific sub-categories for pedestrian, and thus an Inception Score model trained on a generic image recognition dataset (Zhou et al. 2019) may fail to capture the quality and diversity of pedestrian generation results. To this end, we propose two evaluation metrics, named Pose Score and Pose Variance, grading the generation quality and generation variety simultaneously. Based on a Pose Estimation model pre-trained on a larger pedestrian dataset, the evaluation is easy to compute in practice.

To summarize, this paper makes the following key contributions:

- We extend previous fine-grained text-to-image synthesis research by advocating a more challenged pedestrian generation problem.

- We proposed a novel MGD-GAN model that exploits a visual-semantic attention enhanced human-part-based Discriminator as well as a self-cross-attended global Discriminator, alleviating the unbalance competition bottleneck between Discriminator and Generator.

- We present two novel pedestrian generation metrics, named pose score and pose variance, as a non-trivial complement to existing ones.

- The proposed MGD-GAN model achieves the best results on the challenging CUHK-PEDES dataset. The extensive ablation studies verify the effectiveness of each component within the MGD-GAN model in different aspects, i.e., generation quality, generation variety, and the consistency with the textual description.

\section{Related Works \\ Person image generation}

Previous person image generation methods mainly focus on the pose-transfer task, which aims to generate person images with the expected poses (Ma et al. 2017; Ma et al. 2018; Siarohin et al. 2018, Grigorev et al. 2019: Zhu et al. 2019b). Recently, Zhou et al.(Zhou et al. 2019) presents a two-stage model to manipulate the visual appearance (pose and attribute) of a person image according to natural language descriptions. Despite this progress, our work synthesizes a person image based solely on the textual descriptions, which is a more challenging task and has different applications.

\section{Text-to-image generation}

The challenging and open-ended nature of text-to-image generation lends itself to a variety of diverse models. There are mainly two lines of works. The first is generating complex scenes with multiple objects (Hong et al. 2018 Li et al. 2019; Hinz, Heinrich, and Wermter 2019). The other is the generation on fine-grained categories (Wei, $\mathrm{Wu}$, and Cui 2019). In this line of works, GAN-INT-CLS (Reed et al. 2016) firstly learns a joint embedding of text and images to capture the fine-grained relationship between them. After that, a conditional GAN is introduced to learn the mapping from text embeddings to images. Zhang et al.(Zhang et al. 2017) stacks multiple generators to remedy the fine-grained details from previous generators. HD-GAN (Zhang, Xie, and Yang 2018) uses hierarchically-nested discriminators to generate photographic images. More recently, the AttnGAN (Xu et al. 2018) can synthesize fine-grained details of subtle regions by performing an attention equipped generative network. The main difference between our method and previous arts is two-fold. First, we advocate solving a more challenging text-to-pedestrian generation problem besides the existing birds/flowers generation. Second, we present the globallocal attentional discriminators to ease the unbalanced adversarial training problem within previous works.

\section{Multi-Grained Discrimination enhanced Generative Adversarial Network}

In this section, we will elaborate on each building block comprising the MGD-GAN model. As shown in Figure 2. MGD-GAN mainly embodies three modules: the multistage attentional generator, the visual-semantic attention enhanced human-part-based discriminator, and the self-crossattended global discriminator.

Technically, given the sentence feature $s$ and words feature $W$, we want to synthesize the desired human picture $I$. The sentence feature $s \in \mathbb{R}^{N_{s}}$ and the words feature $W=\left\{w_{1}, w_{2}, \ldots, w_{T}\right\}, w_{t} \in \mathbb{R}^{N_{w}}$ are pre-trained using a cross-modal representation learning network named DAMSM (Xu et al. 2018). $T$ is the specified max sequence length of sentences, $N_{s}$ is the dimension of sentence features and $N_{w}$ is the dimension of words features.

\section{Multi-stage generation strategy}

We follow a standard multi-stage baseline (Zhang et al. 2018b; Zhu et al. 2019a) to develop our generator. Rather than creating a high-resolution picture directly from the textual description $s$, we simplify the generation process by first creating a low-resolution picture $I_{0}$, which relies on only rough shapes and colors. To remedy fine-grained errors, we incorporate a refinement generator $G_{i+1}$ which is conditioned on the low-resolution outcomes $I_{i}$ and the words features $W$. The first generator $G_{0}$ and multiple refinement 

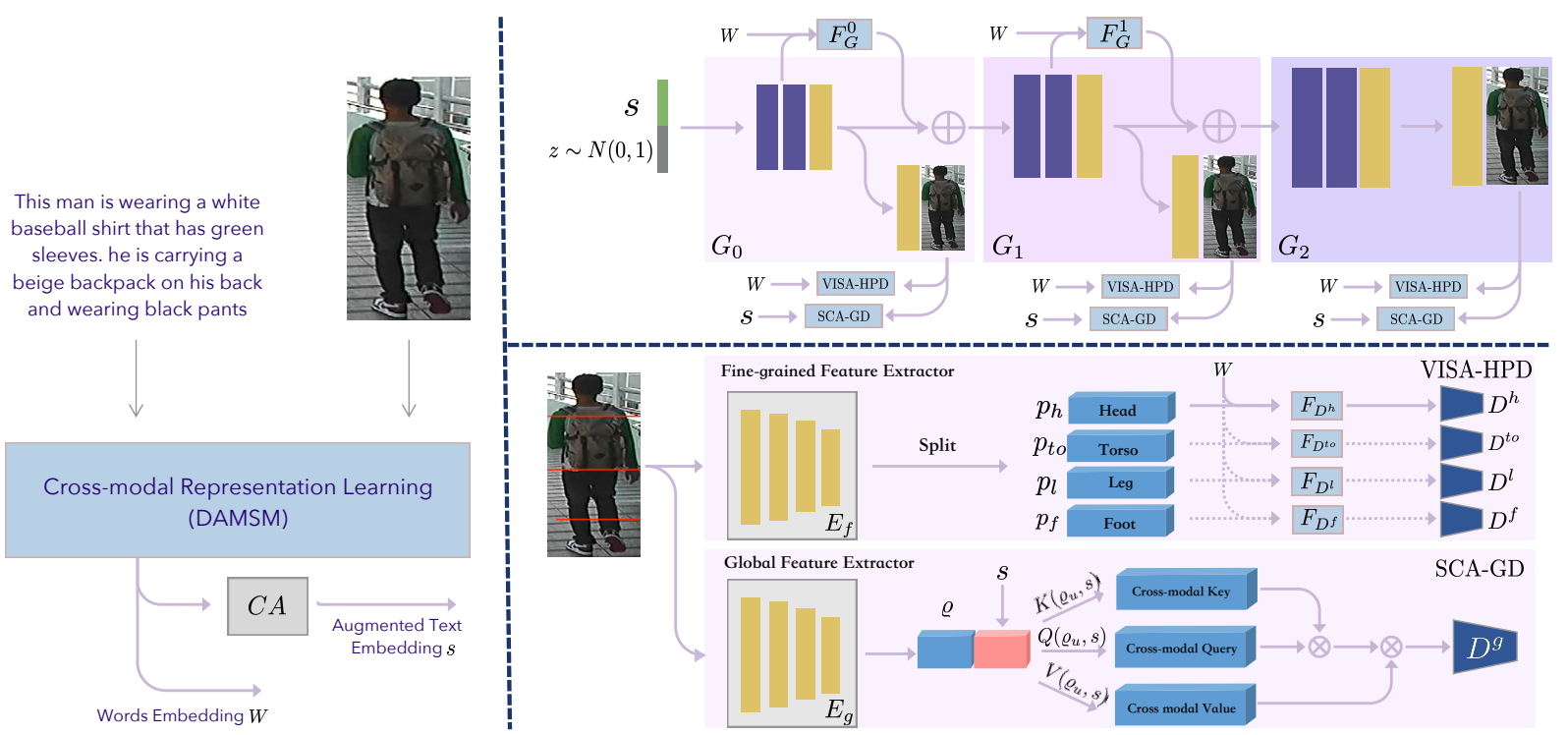

Figure 2: The overall scheme of our proposed MGD-GAN model. The initial text embedding $s$ and words embeddings $W$ are obtained by a pre-trained cross-modal representation learning model named DAMSM. At each training stage $i$, we build a human-part-based discriminator VISA-HPD functioning at the words-regions level and a global discriminator SCA-GD at the sentence-image level, as well as a fine-grained generator.

generators $\left\{G_{1}, \ldots, G_{m-1}\right\}$ can be stacked sequentially and form the multi-stage generation process.

In order to enforce the model to produce fine-grained details within the words feature (Xu et al. 2018) and carry out compositional modeling (Cheng et al. 2019), we construct several attention modules $F_{G}^{i}$ for adjacent generators $\left(G_{i}, G_{i+1}\right)$. The structure of $F_{G}^{i}$ is the same as VISA.

\section{VISA-HPD: Visual-Semantic Attention enhanced Human-Part-based Discriminator}

To leverage some domain knowledge for the fine-grained text-to-pedestrian problem, we investigate some popular methods used in person re-identification research field and propose a human-part-based approach. The core idea in the behind is simple but effective. In order to tackle the complex structure of the human body, we split the encoded feature $\rho$ from the generated image $I$ vertically and equally into several parts, i.e., the head part $p_{h}$, the torso part $p_{t o}$, the leg part $p_{l}$ and the foot part $p_{f}$. The correctness, the coherence, and the faithfulness to the corresponding words and phrases of each human part are individually scored by a related discriminator, i.e., $D^{\kappa}, \kappa \in\{h, t o, l, f\}$. We note that human part discriminators share a common fine-grained feature encoder $E_{f}$ but do not share parameters.

Previous GAN-based models for fine-grained text-toimage generation typically equip the Generator with attention mechanisms. In order to grade the relevance of regions and words, we develop a Visual-Semantic Attention, named VISA, for each human part discriminator. The VISA module $F_{D^{\kappa}}$ takes the words features $W \in \mathbb{R}^{N_{w} \times T}$ and the human part regions features $p_{\kappa} \in \mathbb{R}^{N_{r} \times N / 4}$ as input. $N$ is the number of regions in $\rho$ and $N_{r}$ is the dimension of regions.
For each image region feature $\rho_{u}$, we compute the attention weights with words features $W$ using a FullyConnected layer $f$ and a softmax layer. We obtain the final words-attended region feature as the addition of original region feature and the weighted sum of words features.

$$
\begin{aligned}
\alpha_{u, t} & =\frac{\exp \left(f\left(\rho_{u}, w_{t}\right)\right)}{\sum_{t} \exp \left(f\left(\rho_{u}, w_{t}\right)\right)} \\
\rho_{u}^{\prime} & =\sum_{t} \alpha_{u, t} w_{t} \\
r_{u} & =\rho_{u}+\rho_{u}^{\prime}
\end{aligned}
$$

Hence, the discrimination process for generated image $I$ can be expressed as:

$$
\begin{aligned}
& \rho=E_{f}(I) \\
& p_{h}, p_{t o}, p_{l}, p_{f}=\operatorname{Split}(\rho) \\
& y_{\kappa}=D^{\kappa}\left(F_{D^{\kappa}}\left(W, p_{\kappa}\right)\right)
\end{aligned}
$$

where $y_{\kappa} \in(0,1)$ is the output of each human part discriminator, which grades the generation quality and faithfulness to attended words of each human part.

\section{Self-cross-attended Global Discriminator}

As a non-trivial counterpart of the local discriminator stated above, a self-cross-attended global discriminator is proposed to capture the coherence of the body structure as a whole. Extending beyond the Self-Attention GAN (Zhang et al. 2018a), we not only build a self-attention map of spatial context but also harness cross-modal context at the sentenceimage level. Specifically, we build the self-cross-attention scores of cross-modal context by: 


$$
\beta_{v, u}=\frac{\exp \left(c_{u v}\right)}{\sum_{u=1}^{N} \exp \left(c_{u v}\right)}, \text { where } c_{u v}=K\left(\varrho_{u}, s\right)^{T} Q\left(\varrho_{v}, s\right)
$$

where $\varrho_{u}, \varrho_{v} \in \mathbb{R}^{N_{r}}$ denote region features within $\varrho=$ $E_{g}(I)$ extracted by a global feature extractor $E_{g} . K$ and $Q$ are joint image-sentence feature spaces, formulated as.

$$
K\left(\varrho_{u}, s\right)=W_{k}\left(\left[\varrho_{u}, s\right]\right)
$$

Therefore, $\beta_{v, u}$ indicates to what magnitude the $u^{\text {th }}$ image region is attended to synthesize the $v^{t h}$ image region concerning the semantic context at the sentence level. The final self-cross-attention map $o=\left(o_{1}, o_{2}, \ldots, o_{v}, \ldots, o_{N}\right) \in$ $\mathbb{R}^{N_{r} \times N}$ can be obtained by:

$$
o_{v}=W_{z}\left(\sum_{u=1}^{N} \beta_{v, u} V\left(\varrho_{u}, s\right)\right)
$$

where $V$ is another $1 \mathrm{x} 1$ convolution besides $K$ and $Q$.

\section{Objective Functions}

The objective function of our MGD-GAN is composed of three parts, i.e., the adversarial loss, the conditioning augmentation loss, and the DAMSM loss.

Adversarial Loss A common practice is to employ two adversarial losses: the unconditional adversarial loss and the conditional visual-semantic adversarial loss. We further expand the conditional visual-semantic adversarial loss hierarchically, i.e., a fine-grained words-regions adversarial loss and a global sentence-image adversarial loss.

The generator $G$ and discriminator $D$ are alternatively trained at each learning stage of MGD-GAN. In particular, the adversarial loss of the generator $G_{i}$ at the $i^{\text {th }}$ stage can be defined as:

$$
\begin{aligned}
\mathcal{L}_{G_{i}}^{a d v} & =-1 / 3[\underbrace{-\mathbb{E}_{I_{g} \sim p_{G_{i}}} \log D_{i}^{g}\left(I_{g}\right)}_{\text {unconditional loss }}+\underbrace{\mathbb{E}_{I_{g} \sim p_{G_{i}}} \log D_{i}^{g}\left(I_{g}, s\right)}_{\text {global conditional loss }} \\
& +\underbrace{1 / 4 \sum_{\kappa} \mathbb{E}_{I_{g} \sim p_{G_{i}}} \log D_{i}^{\kappa}\left(I_{g}, W\right)}_{\text {local conditional loss }}]
\end{aligned}
$$

where the $D_{i}^{g}$ stands for the self-cross-attended global discriminator. The unconditional loss is intended to distinguish the generated image from the real image. The global conditional loss and local conditional loss are designed to determine whether the generated image is faithful to the input description at the sentence-image level and the wordsregions level, respectively.

We define the loss function of two discriminators as:

$$
\begin{aligned}
& \mathcal{L}_{D_{i}^{g}}=-\frac{1}{2}\left[\mathbb{E}_{I_{d} \sim p_{\text {data }}} \log D_{i}^{g}\left(I_{d}\right)+\mathbb{E}_{I_{g} \sim p_{G_{i}}} \log \left(1-D_{i}^{g}\left(I_{g}\right)\right)\right. \\
& \left.\quad+\mathbb{E}_{I_{d} \sim p_{\text {data }}} \log D_{i}^{g}\left(I_{d}, s\right)+\mathbb{E}_{I_{g} \sim p_{G_{i}}} \log \left(1-D_{i}^{g}\left(I_{g}, s\right)\right)\right]
\end{aligned}
$$

$\mathcal{L}_{D_{i}^{\kappa}}=-\mathbb{E}_{I_{d} \sim p_{\text {data }}} \log D_{i}^{\kappa}\left(I_{d}, W\right)-\mathbb{E}_{I_{g} \sim p_{G_{i}}} \log \left(1-D_{i}^{\kappa}\left(I_{g}, W\right)\right)$

It is worth noting that the loss function of the human-part discriminator $\mathcal{L}_{D_{i}^{\kappa}}$ does not contain an unconditional adversarial loss. We assume that a typical conditional adversarial loss does measure the visual quality of images as well as the visual-semantic correspondence and that the body structure is better captured as a whole.

Conditioning Augmentation Loss To mitigate the discontinuity problem caused by limited training data, we corporate a regularization term, named Conditioning Augmentation (Zhang et al. 2017), formulated as follows:

$$
\mathcal{L}_{\text {cond }}=D_{K L}(\mathcal{N}(\mu(s), \Sigma(s)) \| \mathcal{N}(0, \mathrm{I}))
$$

where $D_{K L}$ represents the Kullback-Leibler divergence. The estimation of mean $\mu(s)$ and diagonal covariance $\Sigma(s)$ are modeled by fully-connected layers.

DAMSM Loss As a common practice (Xu et al. 2018, Cheng et al. 2019; Zhu et al. 2019a), we employ a cross-modal representation learning based module named DAMSM. This module provides the initial sentence and words embeddings, as well as a matching loss $\mathcal{L}_{D A M S M}$ of the generated image and conditioned text.

Therefore, the final loss function of the generator can be written as:

$$
\mathcal{L}_{G_{i}}=\sum_{i} \mathcal{L}_{G_{i}}^{a d v}+\lambda_{1} \mathcal{L}_{\text {cond }}+\lambda_{2} \mathcal{L}_{D A M S M}
$$

\section{Pose Score \& Pose Variance}

In current literature, the numerical assessment approaches for GANs are not explicitly intended for pedestrian generation, such as Inception Score.

Under the observation that pedestrian has no salient subcategories like birds and flowers, the classification model for Inception Score trained on a generic image recognition dataset may fail to evaluate the generation quality and diversity competently. To this end, we propose two pedestrianspecific generation metrics named Pose Score and Pose Variance as a non-trivial complement to the Inception Score.

Specifically, we first pre-train a pose estimation model (Cao et al. 2017) on a larger COCO 2016 keypoints challenge dataset (Lin et al. 2014). For each generated pedestrian $I_{\epsilon}, \epsilon \in\{1, \ldots, \Xi\}$, we detect the 2D positions of body parts, represented as $B_{\epsilon}=\left\{b_{\epsilon, 1}, b_{\epsilon, 2}, \ldots, b_{\epsilon, \tau}, \ldots, b_{\epsilon, J}\right\}$ where $b_{\epsilon, \tau} \in \mathbb{R}^{2}$, one per part. Some parts may not be detected because the part is occluded or blurred. Under the assumption that there is no occlusion in the CUHK-PEDES dataset, we use the average ratio of the number of detected parts and the upper-bound, i.e., $P S=\frac{1}{\Xi} \sum_{\epsilon} \frac{\# B_{\epsilon}}{18}$, as the Pose Score.

As for Pose Variance, we individually compute the variance of each body part for all generated pedestrians and average them. The intuition is that the larger the variance of 


\begin{tabular}{l|ccccccc}
\multicolumn{1}{c|}{ Method } & BLEU-1 & BLEU-2 & BLEU-3 & BLEU-4 & METEOR & ROUGE_L & CIDEr \\
\hline \hline Reed et al. (Reed et al. 2016) & 0.496 & 0.321 & 0.218 & 0.151 & 0.211 & 0.454 & 0.949 \\
StackGAN (Zhang et al. 2017) & 0.512 & 0.337 & 0.233 & 0.163 & 0.219 & 0.468 & 1.125 \\
AttnGAN (Xu et al. 2018) & 0.561 & 0.396 & 0.293 & 0.222 & 0.253 & 0.525 & 1.519 \\
\hline \hline Ours & $\mathbf{0 . 5 6 5}$ & $\mathbf{0 . 4 0 1}$ & $\mathbf{0 . 2 9 9}$ & $\mathbf{0 . 2 2 9}$ & $\mathbf{0 . 2 5 7}$ & $\mathbf{0 . 5 3 0}$ & $\mathbf{1 . 5 5 3}$ \\
\hline Real images (upper bound) & 0.599 & 0.443 & 0.340 & 0.268 & 0.282 & 0.565 & 1.855 \\
\hline
\end{tabular}

Table 1: Quantitative evaluation results of caption generation on different natural language generation metrics. The last row presents the performance of real images, which can be viewed as the upper-bound of the evaluation metrics.

human poses is, the more diversified pedestrians the model can generate. Mathematically, the proposed Pose Variance can be computed as:

$$
P V=\exp \left(\frac{1}{J * 2} \sum_{\tau=1}^{J} \sum_{\iota=1}^{2} \operatorname{Var}\left(\left\{\frac{b_{\epsilon, \tau, \iota}}{b_{\max }}\right\}_{\epsilon=1}^{\Xi}\right)\right)
$$

where $b_{\max }$ is a normalization factor and is set to 256 , which is the width or height of generated image $I$.

\section{Experiments}

\section{Experiment setup}

Dataset As far as we know, the CUHK-PEDES dataset ( $\mathrm{Li}$ et al. 2017) is the only caption-annotated pedestrian dataset. It contains 40,206 images over 13,003 persons. Images are collected from five existing person re-identification datasets, CUHK03 (Li et al. 2014), Market-1501 (Zheng et al. 2015) , SSM (Xiao et al. 2016), VIPER (Gray, Brennan, and Tao 2007), and CUHK01 (Li, Zhao, and Wang 2012) while each image is annotated with 2 text descriptions by crowdsourcing workers. Sentences incorporate rich details about person appearances, actions, poses. The nature of abundant vocabulary and little repetitive information makes it a better dataset for the fine-grained text-to-image generation. We follow the original dataset split, which includes 34,054 images for training, 3,078 for validation, and 3,074 for testing.

Evaluation metric We choose to evaluate the generation result using various metrics, i.e., Inception Score, our newly proposed Pose Score \& Variance, and Caption Generation.

Inception Score - Following standard practice, we use a pre-trained Inception v3 network to compute the Inception Score (Salimans et al. 2016). Inception Score measures the visual quality and the generation diversity and has been known to be correlated with human perceptions.

Pose Score \& Pose Variance - Our newly proposed Pose Score/Variance metrics, which separately measure the visual quality of different body parts and generation diversity concerning human poses.

Caption Generation - In order to reflect whether the generated image is well-conditioned on input text, we adopt a caption generation based approach (Hong et al. 2018). The intuition behind this approach is that if the generated image is faithful to input text, a well-trained caption model on the same dataset can reconstruct the input text accordingly. We use this caption architecture (Xu et al. 2015) and

\begin{tabular}{l|ccc}
\multicolumn{1}{c|}{ Models } & IS & PS & PV \\
\hline \hline Reed et al. & $4.32 \pm 0.194$ & 0.275 & 1.935 \\
\hline StackGAN & $4.79 \pm 0.184$ & 0.362 & 1.945 \\
\hline AttnGAN & $5.07 \pm 0.396$ & 0.465 & 2.039 \\
\hline MGD-GAN & $\mathbf{5 . 7 4} \pm \mathbf{0 . 5 2 6}$ & $\mathbf{0 . 4 8 9}$ & $\mathbf{2 . 0 5 3}$
\end{tabular}

Table 2: Quantitative evaluation results on generation visual quality. IS, PS and PV stands for the Inception Score and the proposed Pose Score/Variance metrics. The PS and PV of real images are 0.774 and 2.388 .

this implementation 1 trained on the CUHK-PEDES dataset. We measure the similarity of generated captions and input text using four standard Natural Language Generation metrics, BLEU (Papineni et al. 2002), METEOR (Banerjee and Lavie 2005), ROUGE_L (Lin 2004) and CIDEr (Vedantam, Lawrence Zitnick, and Parikh 2015).

Implementation Details As stated in the previous section, we obtain the sentence embeddings and words embeddings from DAMSM and fix them during training. We have $m=3$ training stages which generate images of resolutions $64 \times 64$, $128 \times 128$ and $256 \times 256$ individually. We set $N_{w}=256$ and $N_{r}=512$ as the dimension of words features and intermediate region features. The hyperparameter of generator loss is set as $\lambda_{1}=1$ and $\lambda_{2}=5$. The dimension of augmented sentence embedding $N_{s}$ is set to 100 .

\section{Quantitative evaluation}

As far as we know, we are the initiative to do text-topedestrian generation. We compare our method with several works for text-to-bird/flower generation.(Reed et al. 2016 Zhang et al. 2018b; Xu et al. 2018).

Overall, the results across multiple evaluation metrics on the CUHK-PEDES test dataset consistently indicate that our proposed MGD-GAN achieves better results against the other three methods, concerning both the visually authenticity and text-image consistency.

Specifically, the Inception Score, Pose Score and Pose Variance of MGD-GAN and other methods are in Table 2 MGD-GAN achieves the best results across these three metrics. Compared with the state-of-art method on fine-grained text-to-image generation, the AttnGAN (Xu et al. 2018),

\footnotetext{
${ }^{1}$ https://github.com/sgrvinod/a-PyTorch-Tutorial-to-ImageCaptioning
} 

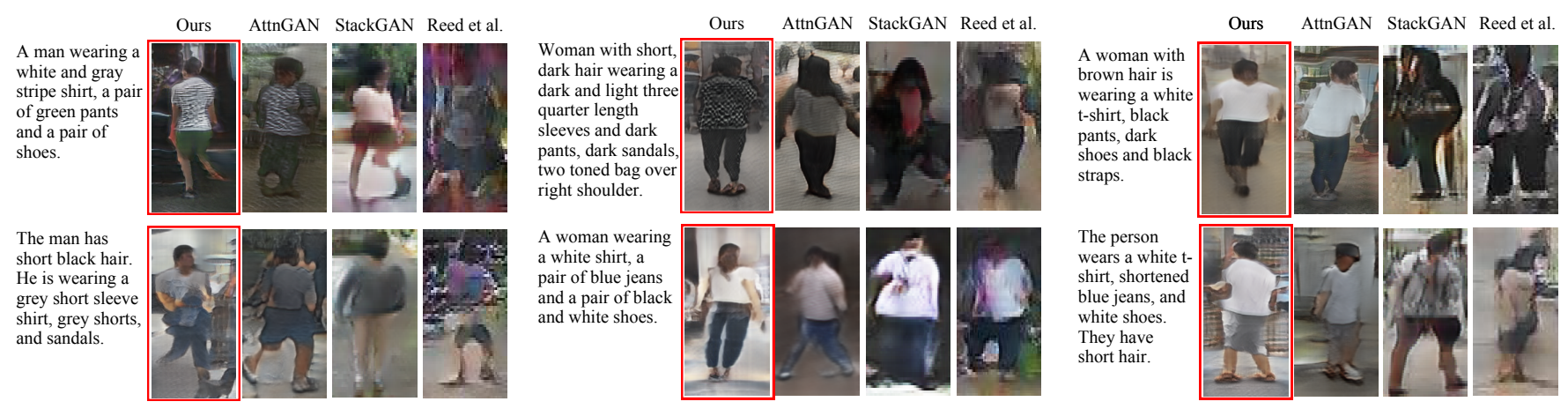

Figure 3: Qualitative comparison of four methods. Generated images are conditioned on text descriptions from CUHK-PEDES test set. The image generated by our method is listed in the first column for every example and is boxed off in red. Despite some failure of small objects like straps, our method outperforms other methods qualitatively in most cases.

MGD-GAN improved the Inception Score from 5.07 to 5.74, the Pose Score from 0.465 to 0.489 and the Pose Variance from 2.039 to 2.053 . These results indicate that the proposed modules in MGD-GAN are promising directions for the fine-grained text-to-image generation.

As for the evaluation of whether the generated images are well-conditioned on the input text, the natural language generation evaluation results on the generated texts are listed in Table 1 Our MGD-GAN achieves better results in terms of all the seven evaluation metrics.

\section{Qualitative evaluation}

Figure 3 demonstrates the synthesized images produced by our MGD-GAN and three state-of-the-art models in the context of quality assessment. All samples are conditioned on text descriptions on CUHK-PEDES test set. Our MGDGAN method produces pedestrians with a coherent structure and vivid details in most cases, comparing to the AttnGAN, the StackGAN and the Reed et al.. For convenience, we use $C_{i j}$ to refer the $i_{t h}$ row and the $j_{t h}$ column example.

Subjective analysis Due to the lack of attention mechanism designed for fine-grained image generation, StackGAN and Reed et al.generate images with vague appearance $\left(C_{12}, C_{13}\right)$ and inconsistent body structure $\left(C_{11}, C_{22}\right)$. The AttnGAN method, which employs an attention mechanism through the generator, achieves better results. For example, the $C_{13}$ generated by AttnGAN looks much more realistic and has most details described by the text, including "white t-shirt" and "black pants". By comparing MGD-GAN with AttnGAN, we can see that MGD-GAN further improves many fine-grained details, such as the facial contour in $C_{21}$ and hairstyle in $C_{22}$, which depicts the femaleness. The result indicates the merit of leveraging fine-grained attention mechanism in both generator and discriminator collaboratively for generating semantically consistent images.

Controllable image generation By altering the colors of wearings, Figure 4 shows the controllable image generation results. Our model can generate pedestrians with fine-

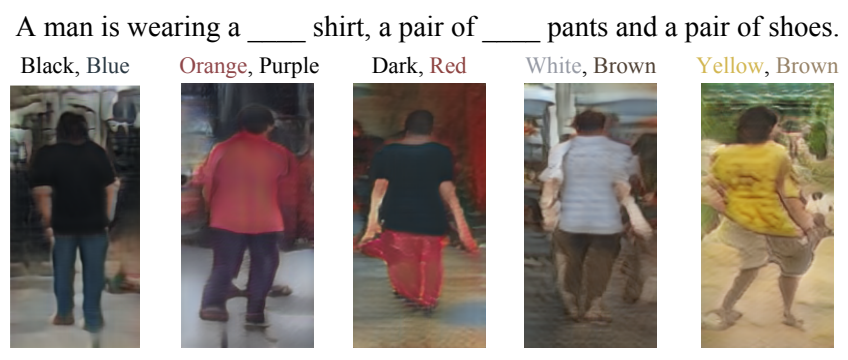

Figure 4: Controllable image generation by deliberately changing color attributes.

grained details authentic to the input text, and diversified poses and appearances. For example, there are pedestrians wearing long pants (the first columns) and short pants (the $5_{t h}$ column), with hands in the pocket (the first column), hands putting in front (the second column) and hands stretching on both sides (the third column).

Multi-stage Refinement \& Attention visualization To better understand the effectiveness of our proposed modules, we visualize the multi-stage generation results and attention maps of the baseline method (without VISA-HPB and SCA-GD) and our method (Figure 5). It can be seen that 1) The MGD-GAN can generate more details than the baseline method, such as the hands and the "white and black shoes". The body structure is also better. 2) With a more balanced training of generator and discriminator, the attended words of the generator in our model are more meaningful and more closely related to human appearance and structure. 3) Our model can progressively refine the body structure, correct irregular artifacts, and remedy missing attributes in images from the previous stages.

\section{Ablation study}

In order to verify the efficacy of different components and gain a better understanding of the network's behavior, we conduct the ablation study by progressively removing control components. The control components are set up to HPD, 
A woman wearing a blue shirt, a pair of blue jeans and a pair of white and black shoes.

Ours

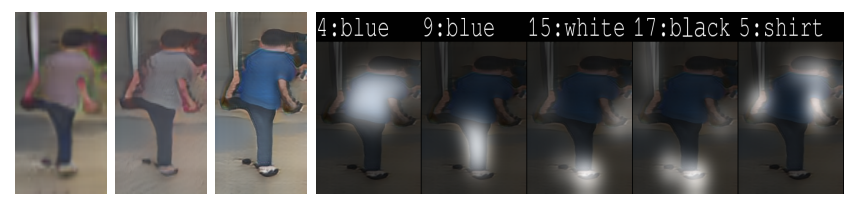

Baseline
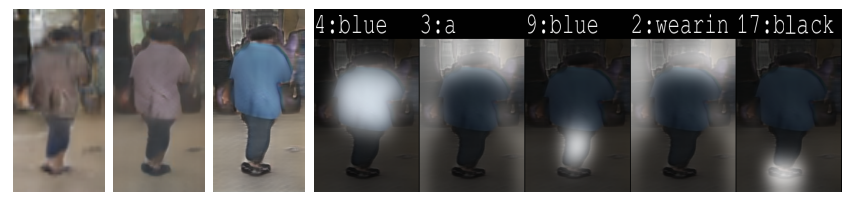

Figure 5: Comparison of the multi-stage generation results and the top 5 relevant words selected by attention module between the proposed method and the baseline method.

\begin{tabular}{l|ccc}
\multicolumn{1}{c|}{ Models } & IS & PS & PV \\
\hline \hline Baseline & $5.12 \pm 0.470$ & 0.275 & 1.979 \\
\hline +HPD & $5.32 \pm 0.357$ & 0.486 & 1.981 \\
\hline +HPD+VISA & $5.59 \pm 0.462$ & $\mathbf{0 . 4 9 8}$ & 2.018 \\
\hline +HPD+VISA+SCA & $\mathbf{5 . 7 4} \pm \mathbf{0 . 5 2 6}$ & 0.489 & $\mathbf{2 . 0 5 3}$ \\
\hline
\end{tabular}

Table 3: Ablation test of different modules in our MGDGAN.

VISA, and SCA, which stands for the human-part-based discriminator, the visual-semantic attention and the self-crossattention respectively. The baseline method, denoted as BL for simplicity, is defined by removing the above modules from the MGD-GAN. The results are shown in Table 3 .

Specifically, the BL achieves a slight performance boost over the AttnGAN by leveraging the VISA in generator instead of the original attention mechanism in AttnGAN. By equipping the human-part-based discriminator, the BL+PD model makes the relative improvement over the BL from 5.12 to 5.32 and 0.470 to 0.486 on Inception Score and Pose Score, respectively. It is worth noting that the VISA enhanced model (BL+PD+VISA) achieves the best performance on Pose Score metric, which verifies the effectiveness of VISA-HPD module in generating fine-grained details of different parts. Moreover, by additionally incorporating a self-cross-attended global discriminator, the MGDGAN model (BL+PD+VISA+SCA) leads to the best performance on the Inception Score (12\% improvement over BL) and Pose Variance metrics.

\section{Person Search Test}

To verify that text-to-pedestrian can help bridge the gap between language and vision and thus promoting many realworld applications, we conduct a text-to-person search experiment the CUHK-PEDES dataset.

We employ the off-the-shelf text-to-image search method named VSE++ (Faghri et al. 2018) as the baseline method. We modify the baseline method by replacing the query from text only to text and an "imagination" picture. The "imagi-

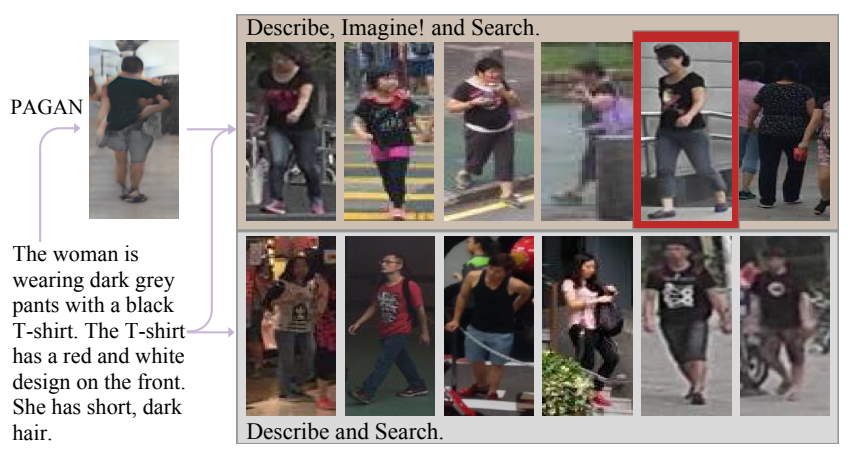

Figure 6: Results of MGD-GAN enhanced search and the baseline method. The image that is boxed of in red is the target image.

\begin{tabular}{l|ccccc} 
Method & $\mathrm{R} @ 1 \uparrow$ & $\mathrm{R} @ 5 \uparrow$ & $\mathrm{R} @ 10 \uparrow$ & Median $\downarrow$ & Average $\downarrow$ \\
\hline \hline Baseline & 18.7 & 46.1 & 58.5 & 7.0 & 51.8 \\
\hline W. Fake & $\mathbf{1 9 . 3}$ & $\mathbf{4 7 . 4}$ & $\mathbf{6 0 . 5}$ & $\mathbf{6 . 0}$ & $\mathbf{4 7 . 2}$ \\
\hline
\end{tabular}

Table 4: The results of generation enhanced person search. Higher is better for recall and lower is better for rank.

nation" is a fake image generated by our MGD-GAN, which is pre-trained on the CUHK-PEDES training set. Original VSE++ contains a text encoder $E n c_{\text {text }}$ and a real image encoder $E n c_{\text {real }}$. We add a fake image encoder $E n c_{\text {fake }}$ which has the same structure as Enc fake. Enc real and $E n c_{f a k e}$ are both pre-trained on ImageNet and fine-tuned during training but do not share weights. Instead of maximizing the similarity of the text representation and the real image representation, we replace the text representation with the joint representation of text and fake image. The joint representation is obtained using a fully connected layer.

The performance comparisons are summarized in Table 4 The consistent improvement over different metrics (Both recall and rank) indicate that our MGD-GAN can help bridge the gap across different modalities and thus enhancing textbased person search. Figure 6 demonstrate a search example. Not only the MGD-GAN enhanced model found the target person image at top 5, but also the retrieved top six images are more relevant in details, such as the design on the front.

\section{Conclusion}

In this paper, we propose the MGD-GAN method for finegrained text-to-pedestrian synthesis. We design a humanpart-based discriminator equipped with the visual-semantic attention, which can be seen as a local discriminator functioning at the words-regions level, as well as a self-crossattended global discriminator at the sentence-image level. We evaluate our model on the caption annotated person dataset CUHK-PEDES. The quantitative results across different metrics, including our proposed Pose Score \& Variance, and substantial qualitative results demonstrate the efficacy of our proposed MGD-GAN method. 


\section{References}

[Banerjee and Lavie 2005] Banerjee, S., and Lavie, A. 2005. METEOR: An automatic metric for MT evaluation with improved correlation with human judgments. In $A C L$.

[Cao et al. 2017] Cao, Z.; Simon, T.; Wei, S.-E.; and Sheikh, Y. 2017. Realtime multi-person $2 \mathrm{~d}$ pose estimation using part affinity fields. In CVPR.

[Cheng et al. 2019] Cheng, Y.; Gan, Z.; Li, Y.; Liu, J.; and Gao, J. 2019. Sequential attention gan for interactive image editing via dialogue. In $C V P R$.

[Faghri et al. 2018] Faghri, F.; Fleet, D. J.; Kiros, J. R.; and Fidler, S. 2018. VSE++: Improving Visual-Semantic Embeddings with Hard Negatives. In $B M V C$.

[Goodfellow et al. 2014] Goodfellow, I.; Pouget-Abadie, J.; Mirza, M.; Xu, B.; Warde-Farley, D.; Ozair, S.; Courville, A.; and Bengio, Y. 2014. Generative adversarial nets. In NIPS.

[Gray, Brennan, and Tao 2007] Gray, D.; Brennan, S.; and Tao, H. 2007. Evaluating appearance models for recognition, reacquisition, and tracking. In Proc. IEEE International Workshop on Performance Evaluation for Tracking and Surveillance (PETS), volume 3, 1-7. Citeseer.

[Grigorev et al. 2019] Grigorev, A.; Sevastopolsky, A.; Vakhitov, A.; and Lempitsky, V. 2019. CoordinateBased Texture Inpainting for Pose-Guided Human Image Generation. In $C V P R$.

[Hinz, Heinrich, and Wermter 2019] Hinz, T.; Heinrich, S.; and Wermter, S. 2019. Generating Multiple Objects at Spatially Distinct Locations. In ICLR.

[Hong et al. 2018] Hong, S.; Yang, D.; Choi, J.; and Lee, H. 2018. Inferring semantic layout for hierarchical textto-image synthesis. In CVPR.

[Li et al. 2014] Li, W.; Zhao, R.; Xiao, T.; and Wang, X. 2014. Deepreid: Deep filter pairing neural network for person re-identification. In $C V P R$.

[Li et al. 2017] Li, S.; Xiao, T.; Li, H.; Zhou, B.; Yue, D.; and Wang, X. 2017. Person Search with Natural Language Description. In $C V P R$.

[Li et al. 2019] Li, W.; Zhang, P.; Zhang, L.; Huang, Q.; He, X.; Lyu, S.; and Gao, J. 2019. Object-driven Text-to-Image Synthesis via Adversarial Training. In CVPR, 12174-12182.

[Li, Zhao, and Wang 2012] Li, W.; Zhao, R.; and Wang, X. 2012. Human reidentification with transferred metric learning. In $A C C V$. Springer.

[Lin et al. 2014] Lin, T.-Y.; Maire, M.; Belongie, S.; Hays, J.; Perona, P.; Ramanan, D.; Dollár, P.; and Zitnick, C. L. 2014. Microsoft coco: Common objects in context. In $E C C V$. Springer.

[Lin 2004] Lin, C.-Y. 2004. Rouge: A package for automatic evaluation of summaries. In $A C L$.

[Ma et al. 2017] Ma, L.; Jia, X.; Sun, Q.; Schiele, B.; Tuytelaars, T.; and Van Gool, L. 2017. Pose guided person image generation. In NIPS.

[Ma et al. 2018] Ma, L.; Sun, Q.; Georgoulis, S.; Van Gool,
L.; Schiele, B.; and Fritz, M. 2018. Disentangled person image generation. In CVPR.

[Papineni et al. 2002] Papineni, K.; Roukos, S.; Ward, T.; and Zhu, W.-J. 2002. BLEU: a method for automatic evaluation of machine translation. In $A C L$.

[Qiao et al. 2019] Qiao, T.; Zhang, J.; Xu, D.; and Tao, D. 2019. MirrorGAN: Learning Text-to-image Generation by Redescription. In CVPR.

[Reed et al. 2016] Reed, S.; Akata, Z.; Yan, X.; Logeswaran, L.; Schiele, B.; and Lee, H. 2016. Generative adversarial text to image synthesis. In $I C M L$.

[Salimans et al. 2016] Salimans, T.; Goodfellow, I.; Zaremba, W.; Cheung, V.; Radford, A.; and Chen, X. 2016. Improved techniques for training gans. In NIPS.

[Siarohin et al. 2018] Siarohin, A.; Sangineto, E.; Lathuilière, S.; and Sebe, N. 2018. Deformable gans for posebased human image generation. In CVPR.

[Vedantam, Lawrence Zitnick, and Parikh 2015] Vedantam, R.; Lawrence Zitnick, C.; and Parikh, D. 2015. Cider: Consensus-based image description evaluation. In CVPR.

[Wei, Wu, and Cui 2019] Wei, X.-S.; Wu, J.; and Cui, Q. 2019. Deep learning for fine-grained image analysis: A survey. arXiv preprint arXiv:1907.03069.

[Xiao et al. 2016] Xiao, T.; Li, S.; Wang, B.; Lin, L.; and Wang, X. 2016. End-to-end deep learning for person search. arXiv preprint arXiv:1604.01850 2:2.

[Xu et al. 2015] Xu, K.; Ba, J.; Kiros, R.; Cho, K.; Courville, A.; Salakhudinov, R.; Zemel, R.; and Bengio, Y. 2015. Show, attend and tell: Neural image caption generation with visual attention. In ICML.

[Xu et al. 2018] Xu, T.; Zhang, P.; Huang, Q.; Zhang, H.; Gan, Z.; Huang, X.; and He, X. 2018. Attngan: Fine-grained text to image generation with attentional generative adversarial networks. In $C V P R$.

[Zhang et al. 2017] Zhang, H.; Xu, T.; Li, H.; Zhang, S.; Wang, X.; Huang, X.; and Metaxas, D. N. 2017. Stackgan: Text to photo-realistic image synthesis with stacked generative adversarial networks. In ICCV.

[Zhang et al. 2018a] Zhang, H.; Goodfellow, I.; Metaxas, D.; and Odena, A. 2018a. Self-attention generative adversarial networks. In ICML.

[Zhang et al. 2018b] Zhang, H.; Xu, T.; Li, H.; Zhang, S.; Wang, X.; Huang, X.; and Metaxas, D. 2018b. Stackgan++: Realistic image synthesis with stacked generative adversarial networks. TPAMI.

[Zhang, Xie, and Yang 2018] Zhang, Z.; Xie, Y.; and Yang, L. 2018. Photographic text-to-image synthesis with a hierarchically-nested adversarial network. In CVPR.

[Zheng et al. 2015] Zheng, L.; Shen, L.; Tian, L.; Wang, S.; $\mathrm{Bu}, \mathrm{J}$.; and Tian, Q. 2015. Person re-identification meets image search. arXiv preprint arXiv:1502.02171.

[Zhou et al. 2019] Zhou, X.; Huang, S.; Li, B.; Li, Y.; Li, J.; and Zhang, Z. 2019. Text Guided Person Image Synthesis. In CVPR. 
[Zhu et al. 2019a] Zhu, M.; Pan, P.; Chen, W.; and Yang, Y. 2019a. Dm-gan: Dynamic memory generative adversarial networks for text-to-image synthesis. In $C V P R$.

[Zhu et al. 2019b] Zhu, Z.; Huang, T.; Shi, B.; Yu, M.; Wang, B.; and Bai, X. 2019b. Progressive Pose Attention Transfer for Person Image Generation. In CVPR. 